\title{
Strain Analysis of Semiconductor Device by Moiré Fringes in STEM Image
}

\author{
Noriaki Endo and Yukihito Kondo
}

EM Business Unit, JEOL Ltd., 3-1-2 Musashino, Akishima, Tokyo 196-8558, Japan.

In scanning transmission electron microscopy (STEM), when the raster spacing is similar to the crystal lattice spacing or the multiple of it and the probe size is smaller than the crystal lattice spacing, the moiré fringes appears due to the under-sampling effect. Since we can arbitrarily select the raster spacing and the rotation angle of the raster direction, we can intentionally make the parallel and/or rotation moiré fringes with wider spacing than the crystal lattice, which is wide enough to recognize even at lower magnification. The moiré fringe bends in regions with a lattice strain in a crystal, and the lattice strain can be measured by the curvature of the fringe, because the spacing and direction of the moiré fringe can be determined by geometrical calculation. D. Su and Y. Zhu observed of a dislocation in a crystal that appears with very large strain, utilizing the STEM moiré fringes method [1]. However, the detection of a small strain, which is utilized for a strained silicon device that has high speed mobility of electrons, has been remained untouched. In this paper, we report the results of the method to detect and quantify the small strain in a practical silicon device over the region wide enough for the device characterization.

The sample and the microscope we used were a 200nm-thick FIB-made semiconductor device and JEM-ARM200F. Figs. 1 (a) and (b) show HAADF images of the device around Si-Ge mixed crystal regions, showing parallel moiré fringes generated with the raster and the lattice fringes due to $\mathrm{Si}$ [220]. Fig. 1 (a) includes the strained region under the gate of the device between diagonal pair of Si-Ge regions. The fringes bend shapely just under the gate of the device, which implies that the region under the gate is strongly strained. In Fig. 1 (b), the fringes gradually bend around Si-Ge crystal. This bend tells us that the strain occurs not only in the region under the gate, but in the wider region of the device around the Si-Ge crystal. The non-strained region located on the right side of each image shows straight moiré fringes, because the region does not contain strain. Thus, the moiré fringes allow us to grasp the distribution of the strain.

By a phase analysis method of holography, the phase map is reproducible from the carrier fringes. Then, if we regard the moiré fringes as carrier fringes of holography, the results after phase analysis on the moiré fringes shows integrated strain map of the Si device. The derivative of the integrated strain map shows the map of strain. In our experiment, the strain of the lattice integrated along the forward direction of horizontal scanning raster. Therefore, the strain map is reproduced in the vertically differentiated map of the integrated strain map. Fig. 2 (a) shows the HAADF image of silicon device showing the tilted moiré fringes. These fringes are rotation moiré fringes caused when scanning raster is tilted with respect to the lattice fringe direction. The moiré fringe in Fig. 2 (a) is generated from the lattice fringes due to $\mathrm{Si}$ [111] and the scanning raster. The raw strain map generally affected by slow and slight displacement of the sample during image acquisition. To reduce this influence, we tried to use the non strained region that is almost simultaneously recorded as a reference. We used the non-strained right quarter region as a reference. Thus, the more accurate strain map can be obtained as a map subtracted from the raw map by the reference map. In Fig. 2 (b), the subtracted strain map is shown. Amount of strain under the gate was measured as a compressive strain of about $1.2 \%$ from the map.

To measure the amount of noise in this method, we performed the strain analysis on the non-strained Si single crystal. The upper panel of Fig. 3 shows HAADF image in left and its strain map in right. The lower figure of Fig. 3 shows the profile of the strain map that indicates the error range of this method. The strain accuracy is estimated to be about $\pm 0.5 \%$ at the present stage. However, the accuracy could be improved by better contrast of the moiré fringes and less noise with 
the averaged map. In conclusion, the strain distribution of a crystal can be reproduced from only a low-magnification STEM image in a wide field which is useful for strained device characterization.

Reference:

[1] D. Su and Y. Zhu, Ultramicroscopy 110(3) (2010), p.229.
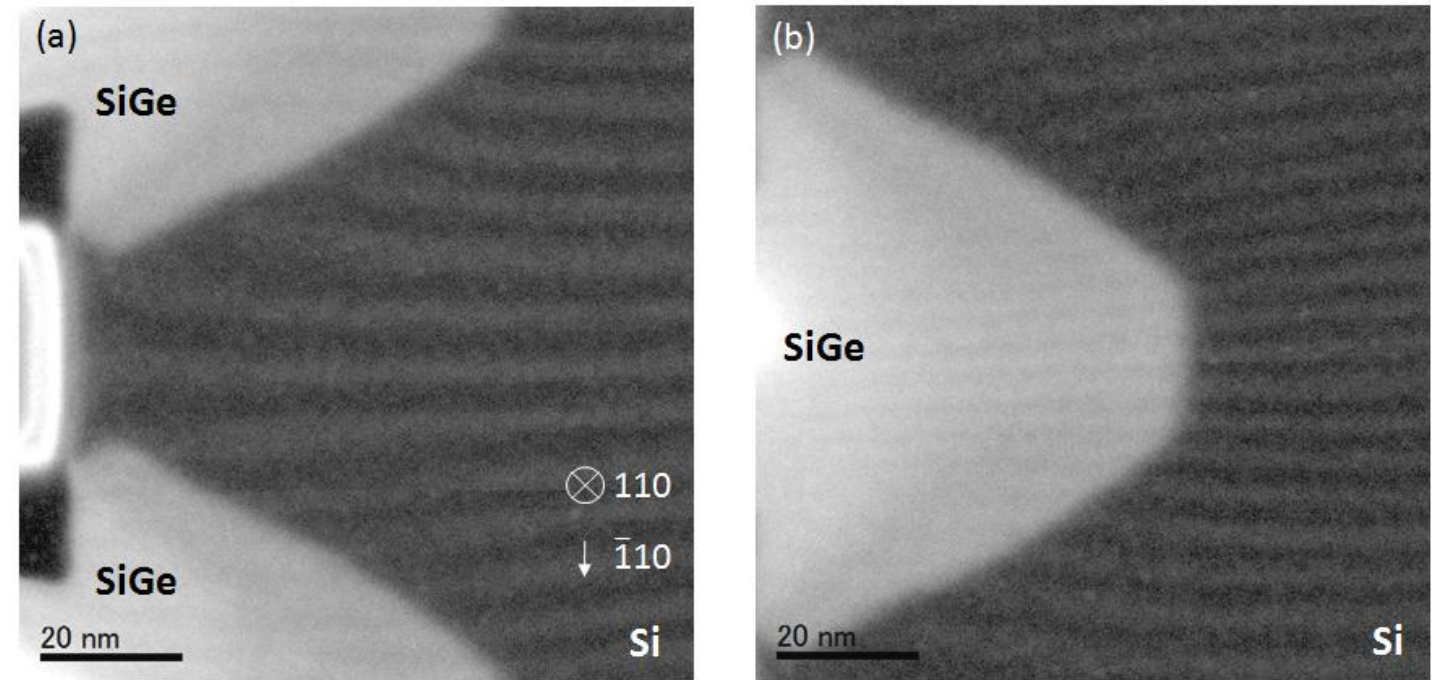

Fig. 1. STEM moiré fringes of Si crystal under the gate (a) and Si-Ge crystal (b).
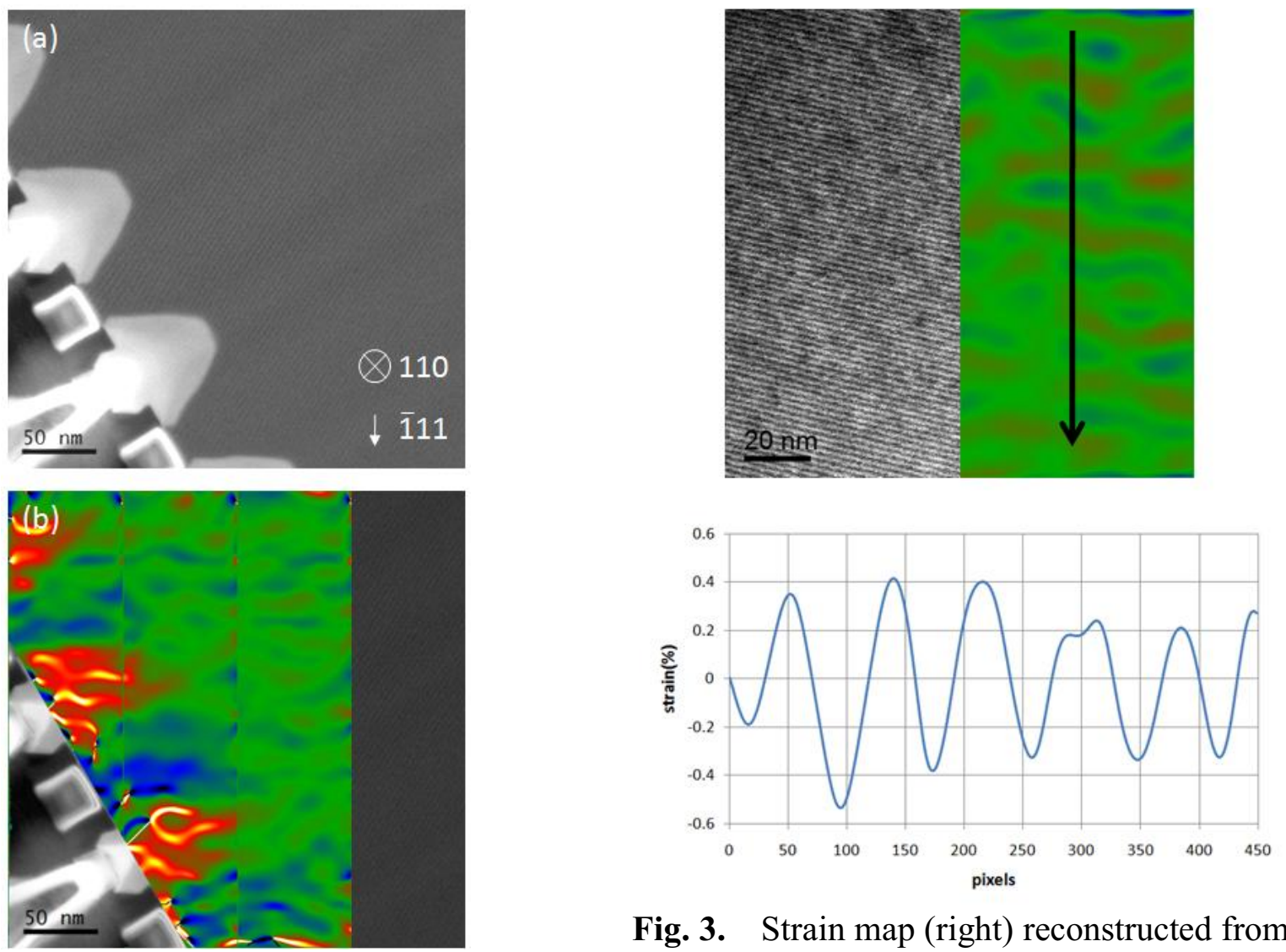

Fig. 3. Strain map (right) reconstructed from

Fig. 2. Strain map (b) reconstructed from STEM moiré fringes (a) of a semiconductor device. STEM moiré fringes (left) of a $\mathrm{Si}$ single crystal without strain and the profile of strain intensity on the arrow of this strain map. 